\title{
D-branes
}

\section{in the background of NS fivebranes}

\author{
Shmuel Elitzur ${ }^{a}$, Amit Giveon $^{a}$, David Kutasov $^{b}$, Eliezer Rabinovici $^{a}$, and Gor Sarkissisan ${ }^{a}$ \\ ${ }^{a}$ Racah Institute of Physics, The Hebrew University, \\ Jerusalem 91904, Israel \\ ${ }^{b}$ Department of Physics, University of Chicago \\ 5640 S. Ellis Av., Chicago, IL 60637, USA
}

Abstract: We study the dynamics of $D$-branes in the near-horizon geometry of $N S$ fivebranes. This leads to a holographically dual description of the physics of $D$-branes ending on and/or intersecting $N S 5$-branes. We use it to verify some properties of such $D$-branes which were deduced indirectly in the past, and discuss some instabilities of non-supersymmetric brane configurations. Our construction also describes vacua of Little String Theory which are dual to open plus closed string theory in asymptotically linear dilaton spacetimes.

\section{Introduction}

The work discussed is based on the results appearing in hep-th/0005052. Due to the restricted space all references should be searched for in the original paper. In the last few years it was found that embedding various supersymmetric gauge theories in string theory, as the low energy worldvolume dynamics on branes, provides an efficient tool for studying many aspects of the vacuum structure and properties of BPS states in these theories. One class of constructions involves systems of $D$-branes ending on and/or intersecting $N S 5$-branes. For applications to gauge theory one is typically interested in taking the weak coupling limit $g_{s} \rightarrow 0$ (as well as the low energy limit). In this limit one might expect the system to be amenable to a perturbative worldsheet treatment, but the presence of the NS5-branes and branes ending on branes complicate the analysis.

In the absence of a derivation of the properties of $D$-branes interacting with $N S 5$-branes from first principles, some of their low energy properties were postulated in the past based on symmetry considerations and consistency conditions. One of the main purposes of this talk is to derive some of these properties by a direct worldsheet study of $D$-branes in the vicinity of $N S 5$-branes.

In the analysis, we will use the improved understanding of the dynamics of $N S 5$-branes achieved in the last few years. It is now believed that in the weak coupling limit (but not necessarily at low energies) fivebranes decouple from gravity and other bulk string modes and give rise to a rich non-gravitational theory, Little String Theory (LST) . It was proposed to study LST using holography. The theory on a stack of NS5branes was argued to be holographically dual to string theory in the near-horizon geometry of the fivebranes. Many properties of LST can be understood by performing computations in string theory in this geometry. In particular, we will find below that it is an efficient way to study properties of $D$-branes ending on or intersecting $N S 5$-branes.

From the point of view of LST, $D$-branes in the vicinity of $N S 5$-branes give rise to new particle and extended object states in the theory, as well as new vacua, typically with reduced supersymmetry (when the branes are space-filling). Another motivation of this work is to understand the non-perturbative spectrum and dynamics of extended objects in LST, and more generally study 
the interplay between the non-trivial dynamics on fivebranes and the worldvolume physics of $D$ branes in their vicinity.

The plan of the talk is the following. In section 2 we review some facts regarding the relevant brane configurations and the physical issues involved. In particular, we describe the conjectured properties of these configurations that we will try to verify. Section 3 is a review of the near-horizon geometry of $N S 5$-branes (the CHS geometry ) and its holographic relation to LST. We also describe a modification of this geometry corresponding to fivebranes positioned at equal distances around a circle, which plays a role in the analysis. In section 4 we review some facts about $D$-branes in flat space and on a threesphere (or $S U(2)$ WZW model). In section 5 we study $D$-branes in the CHS geometry as well as its regularized version. We verify some of the properties described in section 2 , and describe additional features which follow from our analysis.

\section{Some properties of brane config- urations}

One class of brane constructions, that gave rise to many insights into gauge dynamics, involves $D$-branes suspended between $N S 5$-branes. The construction realizes four dimensional $N=1$ supersymmetric gauge theory with gauge group $G=U\left(N_{c}\right)$ and $N_{f}$ chiral superfields in the fundamental representation (more precisely, $N_{F}$ fundamentals $Q^{i}, i=1, \cdots, N_{F}$, and $N_{F}$ anti-fundamentals $\tilde{Q}_{i}$ ). This will help introduce the issues that will be discussed later.

Consider a configuration of $N_{c} D 4$-branes stretched between an NS5-brane and an NS5'brane separated by a distance $L$ along the $x^{6}$ direction (fig. 1). At distances greater than $L$, the five dimensional theory on the $D 4$-branes reduces to a four dimensional theory with $N=1$ supersymmetry. The boundary conditions provided by the fivebranes imply that out of all the massless degrees of freedom on the fourbranes (described by $4-4$ strings), only the $4 d N=1$ vector multiplets for $G=U\left(N_{c}\right)$ survive. To decouple the gauge dynamics from the complications of string theory one takes the limit $g_{s} \rightarrow 0, L / l_{s} \rightarrow 0$, with the four dimensional gauge coupling $g^{2}=g_{s} l_{s} / L$ held fixed.

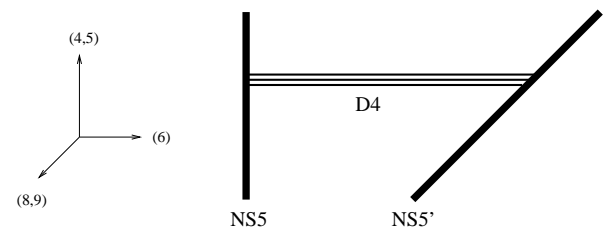

Fig. 1

One can add matter in the fundamental representation of $G$ in one of the two ways illustrated in fig. 2 (which are related). One is to place $N_{f} D 6$-branes between the fivebranes (fig. 2a). The $4-6$ strings give $N_{f}$ fundamentals of $U\left(N_{c}\right), Q^{i}, \tilde{Q}_{i}$, whose mass is proportional to the separation between the sixbranes and the fourbranes in $\left(x^{4}, x^{5}\right)$. Alternatively, one can add to the configuration $N_{f} D 4$-branes stretching from the NS5-brane to infinity (fig. 2b). In this case, the $N_{f}$ fundamentals of $U\left(N_{c}\right)$ should arise from $4-4$ strings stretched between the two kinds of fourbranes. Their mass is the separation between the fourbranes in $\left(x^{4}, x^{5}\right)$. (a)

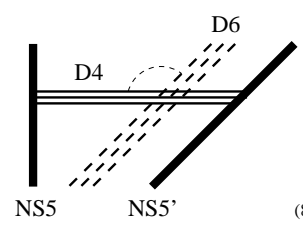

(b)

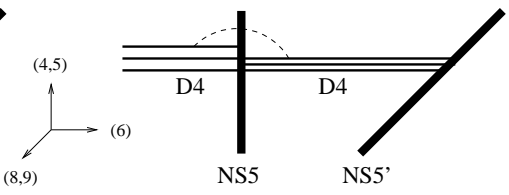

Fig. 2
In the configuration of fig. 2a, the low energy physics should be independent of the locations of the $D 6$-branes along the interval between the fivebranes. It was pointed out that a particularly natural location for the sixbranes is at the same value of $\left(x^{4}, x^{5}, x^{6}\right)$ as the $N S 5^{\prime}$-brane. In this case the $N S 5^{\prime}$-brane is embedded in the $D 6$-branes; it divides them into two disconnected parts (fig. 3). 


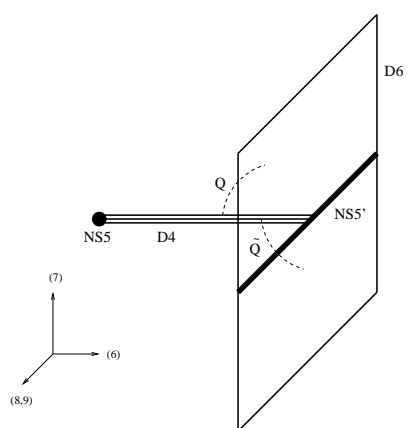

Fig. 3

Consequently, the configuration has two separate $U\left(N_{f}\right)$ symmetries, acting on the two semiinfinite sixbranes, and one may attempt to interpret them as the $U\left(N_{f}\right) \times U\left(N_{f}\right)$ global symmetry of $N=1 \mathrm{SQCD}$, under which $Q$ and $\tilde{Q}$ transform as $\left(N_{F}, 1\right)$ and $\left(1, \bar{N}_{F}\right)$, respectively. This would imply that $4-6$ strings connecting the $D 4$ branes to the upper part of the $D 6$-branes give rise at low energies to the $N_{f}$ chiral multiplets in the fundamental of $U\left(N_{c}\right), Q^{i}$, while those that connect the fourbranes to the lower part of the sixbranes give the $N_{f}$ chiral superfields in the anti-fundamental of $U\left(N_{c}\right), \tilde{Q}_{i}$, as indicated in fig. 3 .

The low-lying excitations of the brane configurations discussed above can be divided into two classes: those that are bound to one of the fivebranes, and those that are not. In this talk we will analyze the properties of the first class of excitations. It includes the following:

- (a) 4-6 strings connecting $N_{c} D 4$-branes to $N_{F} D 6^{\prime}$-branes, all of which end on an NS5-brane ${ }^{1}$ (fig. 4a). The prediction is that they give rise to a chiral spectrum: a chiral superfield $Q$ in the $\left(N_{c}, N_{F}\right)$ of $U\left(N_{c}\right) \times U\left(N_{F}\right)$.

- (b) 4-4 strings connecting fourbranes ending on an NS5-brane from opposite sides (fig. 4b). They should give rise to chiral superfields, $Q$ in the $\left(N_{c}, N_{F}\right)$ and $\tilde{Q}$ in the $\left(\bar{N}_{c}, \bar{N}_{F}\right)$, or hypermultiplets $(Q, \tilde{Q})$.

- (c) 4-6 strings connecting $D 4$-branes ending on NS5-branes to D6-branes intersect-

\footnotetext{
${ }^{1}$ The configuration of fig. 4 a can be obtained from that of fig. 3 by exchanging $\left(x^{4}, x^{5}\right) \leftrightarrow\left(x^{8}, x^{9}\right)$ and removing some branes.
}

ing the fivebranes (fig. 4c). They should also give rise to hypermultiplets $(Q, \tilde{Q})$.

We verify the predictions (a), (b), (c). In this talk we will describe the ingredients leading to the verification of the chiral symmetry.
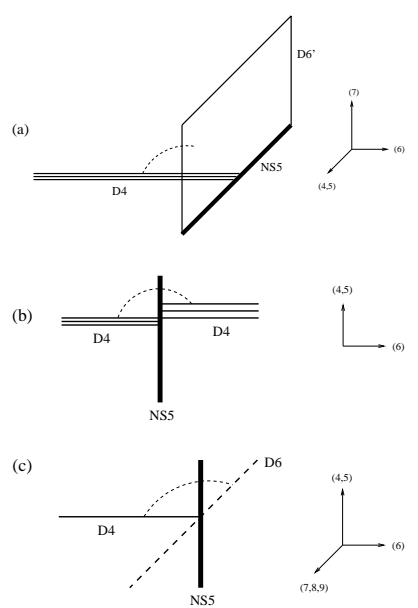

Fig. 4

\section{The near-horizon geometry of NS5- branes and holography}

The background fields around a stack of $k$ parallel NS5-branes are :

$$
\begin{array}{r}
e^{2\left(\Phi-\Phi_{0}\right)}=1+\sum_{j=1}^{k} \frac{l_{s}^{2}}{\left|\vec{x}-\vec{x}_{j}\right|^{2}} \\
G_{I J}=e^{2\left(\Phi-\Phi_{0}\right)} \delta_{I J} \\
G_{\mu \nu}=\eta_{\mu \nu} \\
H_{I J K}=-\epsilon_{I J K L} \partial^{L} \Phi
\end{array}
$$

$I, J, K, L=6,7,8,9$ label the directions transverse to the fivebranes. $\mu, \nu=0,1, \cdots, 5$ are the directions along the brane. $\left\{\vec{x}_{j}\right\}$ are the locations of the fivebranes in $\vec{x}=\left(x^{6}, \cdots, x^{9}\right) . H$ is the field strength of the NS-NS $B$-field; $G, \Phi$ are the metric and dilaton, respectively.

The background (3.1) interpolates between flat ten dimensional spacetime far from the fivebranes, and a near-horizon region, in which the 1 on the right hand side of the first line of (3.1. can be neglected (fig. 5). 


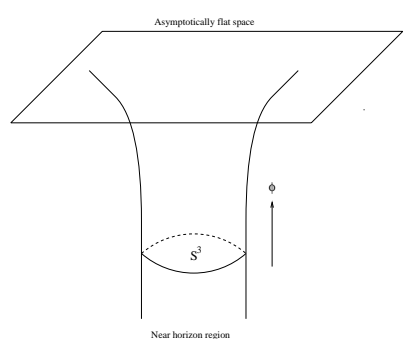

Fig. 5

String propagation in the near-horizon geometry can be described by an exact worldsheet Conformal Field Theory (CFT). The target space is

$$
R^{5,1} \times R_{\phi} \times S U(2)
$$

$R_{\phi}$ corresponds to the radial direction $r=|\vec{x}|$ :

$$
\begin{array}{r}
\phi=\frac{1}{Q} \log \frac{|\vec{x}|^{2}}{k l_{s}^{2}} \\
\Phi=-\frac{Q}{2} \phi
\end{array}
$$

where we set $\Phi_{0}=0$ by rescaling $\vec{x}$. $Q$ is related to the number of fivebranes via

$$
Q=\sqrt{\frac{2}{k}} .
$$

For some reasons it is more convenient to use the coset (cigar) description in this case. The full background now is

$$
R^{5,1} \times \frac{S L(2, R)_{k}}{U(1)} \times \frac{S U(2)_{k}}{U(1)}
$$

Since the background ( ily weakly coupled, one can use it to compute correlation functions in LST in a weak coupling regime in its moduli space of vacua. Consider the (NS,NS) sector of the theory. The observables are primaries of the $N=1$ superconformal algebra with scaling dimension $(h, \bar{h})=\left(\frac{1}{2}, \frac{1}{2}\right)$. By analyzing correlation functions of $V_{j ; m, \bar{m}}$, one finds that poles in correlators correspond to discrete representations of $S L(2)$

$$
|m|=j+n, \quad|\bar{m}|=j+\bar{n} ; \quad n, \bar{n}=1,2,3, \cdots
$$

This leads to a discrete spectrum of states in LST, which exhibits Hagedorn growth at high energy.

\section{Some properties of $D$-branes}

In this section we review some properties of $D$ branes in flat space and on $S^{3}$, in preparation for our discussion of $D$-branes in the CHS geometry $(3.2)$, and its regularized version (3.5.5.

\subsection{4-6 strings in flat space}

Later, we analyze $4-6$ strings connecting $D 4$ branes and $D 6^{\prime}$-branes, both of which end on a stack of $N S 5$-branes (fig. $4 \mathrm{a}$ ).

Consider an open string, one of whose ends is on a $D 4$-brane. The other end is on a $D 6^{\prime}$ brane. The vertex operator describing the (bosonic) ground state of a $4-6^{\prime}$ string stretched between a $D 4$ brane at $\left(x^{8}, x^{9}\right)=(0,0)$ and a $D 6^{\prime}$-brane at $\left(x^{8}, x^{9}\right)=(a, b)$

$$
V=e^{-\varphi} \sigma_{4567} S_{4567} e^{\frac{i}{\pi}\left(a\left(x_{L}^{8}-x_{R}^{8}\right)+b\left(x_{L}^{9}-x_{R}^{9}\right)\right)} e^{i k_{\mu} x^{\mu}}
$$

where $k_{\mu}(\mu=0,1,2,3)$ is the $4 d$ spacetime momentum. $\varphi$ is the bosonized superconformal ghost. The vertex operator (4.1) is written in the -1 picture; one can check that the coefficient of $e^{-\varphi}$ in $(\bar{A} . \overline{1})$ is an $N=1$ superconformal primary, which is a necessary condition for its BRST invariance. The requirement that it has worldsheet dimension $1 / 2$, which is also necessary for BRST invariance, implies that the mass squared of the ground state of the $4-6^{\prime}$ string is $-k_{\mu}^{2}=$ $\frac{1}{\pi^{2}}\left(a^{2}+b^{2}\right)$, as one would expect. (we work in a convention $\alpha^{\prime}=1 / 2$, in which the scalars $x$ are canonically normalized on the boundary and the tension of the fundamental string is $T=1 / \pi$ ). In particular, when the $D 6^{\prime}$-brane intersects the $D 4$-brane, i.e. when $a=b=0$, this mass vanishes. The vertex operator (4.1) describes a particle which transforms as a scalar under $3+1$ dimensional Lorentz rotations. The spin field $S_{4567}$ has 4 components, half of which are projected out by the GSO projection, so (4.1) actually describes two real scalar particles. $\sigma_{4567}$ is a collection of twist fields.

\section{$4.2 D$-branes on the $S U(2)$ group manifold}

We next turn to some facts regarding $D$-branes on a group manifold $G$, focusing on the case $G=$ $S U(2)$. In the absence of $D$-branes, the WZW 
model has an affine $G_{L} \times G_{R}$ symmetry. If $g(z, \bar{z})$ is a map from the worldsheet to the group $G$, the symmetry acts on it as:

$$
g \rightarrow h_{L}(z) g h_{R}(\bar{z})
$$

If the worldsheet has a boundary, there is a relation between left-moving and right-moving modes, and the $G_{L} \times G_{R}$ symmetry is broken. One can still preserve some diagonal symmetry $G$, say the symmetry

$$
g \rightarrow h g h^{-1}
$$

corresponding to $h_{L}=h_{R}^{-1}=h$ in $\left(\begin{array}{l}1 \\ \underline{A} . \overline{2}\end{array}\right)$. The presence of this symmetry constrains the boundary conditions that can be placed on $g$. Allowing $g$ (boundary) $=f$ for some $f \in G$ we must also allow $g$ (boundary) $=h f h^{-1}$ for every $h \in G$. This means that $g$ on the boundary takes value in the conjugacy class containing $f$. For $G=$ $S U(2)$, conjugacy classes are parametrized by a single angle $\theta, 0 \leq \theta \leq \pi$, corresponding to the choice $f=\exp \left(i \theta \sigma_{3}\right)$. Thinking of $S U(2)$ as the group of three dimensional rotations, the conjugacy class $C_{\theta}$ is the set of all rotations of angle $2 \theta$ about any axis. In addition not any value of $\theta$ gives rise to a consistent model. One finds that the possible conjugacy classes on which a boundary state can live are quantized, the corresponding $\theta$ must satisfy

$$
\theta=2 \pi \frac{j}{k}
$$

with $j$ integer or half integer satisfying $0 \leq j \leq$ $\frac{k}{2}$. It is also possible for group element to lie at the boundary in the conjugacy class shifted by arbitrary element $l$ :

$$
g \text { (boundary) } \in C_{\theta} l .
$$

This choice preserves another diagonal subgroup of $(\underline{4} \cdot \overline{4} \cdot \overline{2}): h_{R}=l^{-1} h_{L}^{-1} l$.

\section{D-branes in the near-horizon ge- ometry of $N S 5$-branes}

After assembling the necessary tools, we are now ready to study the physics of the configurations of fig. 4. consider the configuration of fig. 4a. A stack of $N_{c} D 4$-branes ends from the left, i.e. from negative $x^{6}$, on $k$ coincident $N S 5$-branes. $N_{F} D 6^{\prime}$-branes end on the $N S 5$-branes from above (positive $x^{7}$ ). From the point of view of the geometry (3.1.) (fig. 5), the $D$-branes extend into the CHS throat, as indicated in fig. $6 \mathrm{a}$.

The $D 4$-branes intersect the three-sphere at the point $x^{7}=x^{8}=x^{9}=0$; the $D 6^{\prime}$-branes at $x^{6}=x^{8}=x^{9}=0$ (see fig. $6 \mathrm{~b}$ ). Thus, they correspond to the boundary states $\left.g\right|_{\text {boundary }}=1$ and $i \sigma_{3}$, respectively. The $D 4$-branes are described by the boundary state with $\theta=0$ and $l=$ 1 , while the $D 6^{\prime}$-branes correspond to a transformed state, with $\theta=0$ and $l=\exp \left(i \pi \sigma_{3} / 2\right)=$ $i \sigma_{3}$. The $S U(2)$ currents $J^{a}$ satisfy the boundary conditions for strings ending on the $D 4$ and $D 6^{\prime}$ branes, respectively. In order to preserve worldsheet supersymmetry one has to impose analogous boundary conditions on the fermions. For example, for a string ending on the $D 4$-branes one has

$$
\chi^{a}=\bar{\chi}^{a}
$$

while for a boundary on a $D 6^{\prime}$-brane the $\chi^{a}$ satisfy an analog of the sixbranes extend into the throat, the boundary conditions on $\phi$ are Neumann. (a)

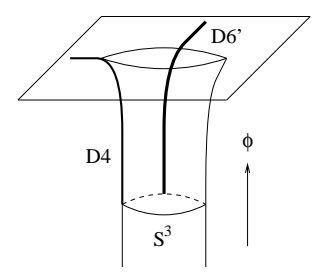

(b)

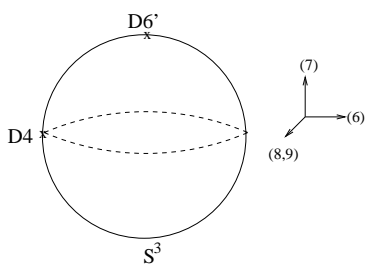

Fig. 6
We would like to construct the vertex operator for emitting the lowest lying $4-6^{\prime}$ string in the geometry of fig. 6 , i.e. generalize (4.1i) to the fivebrane near-horizon geometry. Some parts of the discussion leading to (4.1) are unchanged. In particular, the geometry is the same as there in $\left(x^{0}, x^{1}, x^{2}, x^{3}, x^{4}, x^{5}\right)$. The presence of $\phi$ allows also a contribution $\exp (\beta \phi)$ to the vertex operator. Thus, the analog of $(\stackrel{1}{A} . \overline{1} \overline{1})$ for this case has the form

$$
V=e^{-\varphi} \sigma_{45} S_{45} e^{i k_{\mu} x^{\mu}} e^{\beta \phi} V_{2}
$$


where $V_{2}$ is the contribution of the $S U(2)$ group manifold to the vertex operator, to which we turn next.

The $4-6^{\prime}$ vertex operator $V_{2}$ changes the worldsheet boundary conditions from $g=1$ to $g=l=\exp \left(i \alpha \sigma_{3} / 2\right)$. The $D 6^{\prime}$-brane corresponds to $\alpha=\pi$, but it is instructive to discuss the general case, in which the angle between the $D 4$ and $D 6^{\prime}$-branes is $\alpha / 2$ (see fig. 7 ).

(a)

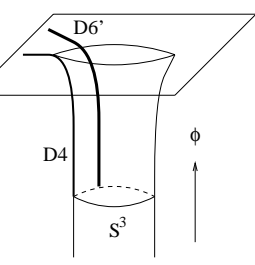

(b)

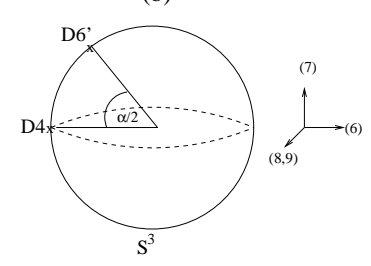

Fig. 7

We find that the lowest lying open string connecting the $D 4$ and $D 6^{\prime}$-branes in the configuration of fig. 7 is described by the vertex operator

$$
V_{4-6^{\prime}}^{+}=e^{-\varphi} \sigma_{45} S_{45} e^{i k_{\mu} x^{\mu}} V_{j m}
$$

with

$$
\frac{k_{\mu}^{2}}{2}+\frac{m^{2}-j(j+1)}{k}=\frac{1}{4}
$$

and

$$
m=-\frac{k}{2} \frac{\alpha}{2 \pi}
$$

To compute the spectrum of low-lying normalizable excitations of the $4-6_{+}^{\prime}$ string, one computes the two point function of the operators $(5.3)$ on the disk. This amplitude exhibits first order poles in $j$. Using the mass-shell condition (5.4), these can be interpreted as poles in $k_{\mu}^{2}$; they correspond to on-shell particles in four dimensions, created from the vacuum by the operator (5.3i).

The calculation of this two point function is very similar to its closed string analog, and is described in appendix A of the original paper. The result is, as in the closed string case, a series of poles corresponding to the discrete representations of $S L(2),(\overline{3} \cdot \bar{\theta})$. The lowest lying state corresponds to $n=1$, i.e. $j=|m|-1$. Plugging this together with $(5.5)$ into $(5.4)$ we find that the mass of the lowest lying normalizable state of the $4-6_{+}^{\prime}$ string is

$$
M^{2}(\alpha)=\frac{1}{2}\left(\frac{\alpha}{\pi}-1\right)
$$

In particular, we find that as expected, for $\alpha=\pi$ the lowest lying state is massless. The vertex operator which creates this massless particle from the vacuum is

$$
V_{4-6^{\prime}}^{+}\left(k_{\mu}^{2}=0\right)=e^{-\varphi} \sigma_{45} S_{45} e^{i k_{\mu} x^{\mu}} V_{\frac{k}{4}-1,-\frac{k}{4}}
$$

The degeneracy works out as well. The chiral structure is recaptured. This validates both the approximate picture and the $L S T$ methods used. We note that for $\alpha<\pi$ the lowest lying state (5.6) is tachyonic; the stable vacuum is obtained by its condensation. This process has a very natural interpretation from the point of view of brane theory, which also makes it clear what is the endpoint of the condensation. For $\alpha \neq \pm \pi$, the configuration of fig. 7 is not supersymmetric, hence stability needs to be checked. For $\alpha<\pi$ the configuration of fig. 7 can reduce its energy by having the $D 4$-brane slide away from the $N S 5$-brane so that it ends on the $D 6^{\prime}$-brane instead (fig. 8). The resulting vacuum is stable. For $\alpha>\pi$, the configuration of figs. 7, 8 is stable under small deformations. Indeed, the lowest lying open string state is massive in this case $(5.6)$.

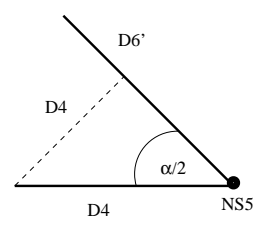

Fig. 8

From the point of view of the brane configurations describing four dimensional gauge theories like that of fig. 3, one can change $\alpha$ from $\pi$ in a number of ways. One is to change the relative position of the $N S 5$ and $N S 5^{\prime}$-branes in $x^{7}$ by the amount $\Delta x^{7}$. In the gauge theory on the $D 4$-branes this corresponds to turning on a Fayet-Iliopoulos D-term. Depending on the sign of the FI term, either $Q$ or $\tilde{Q}$ should condense to minimize the D-term potential

$$
V_{D} \sim\left(Q^{\dagger} Q-\tilde{Q}^{\dagger} \tilde{Q}-r\right)^{2}
$$


where $r$ is proportional to $\Delta x^{7}$. The gauge theory analysis is nicely reproduced by our string theory considerations ${ }^{2} \Delta x^{7}>0$ corresponds to $0<\alpha<\pi$. In this case the ground state of the $4-6+$ string is tachyonic, $Q$ condenses and the $D 4$-branes detach from the $N S 5^{\prime}$-branes and attach to the $D 6$-branes. $\Delta x^{7}<0$ corresponds to $\alpha>\pi$ for the $4-6_{+}$string which is hence massive, but since $0<-\alpha<\pi$ for the $4-6_{-}$ strings $(\tilde{Q})$, a similar process of condensation to the above occurs for them.

Another way of changing $\alpha$ is to tilt the $D 6$ branes by some angle in the $\left(x^{6}, x^{7}\right)$ plane, which must lead to a potential similar to (5.8). The calculations in the cases (b) and (c) mentioned in the introduction can be found in the original paper.

Acknowledgements: This research was supported in part by NSF grant \#PHY94-07194 and by the Israel Academy of Sciences and Humanities - Centers of Excellence Program. The work of A.G. and E.R. is also supported in part by BSF - American-Israel Bi-National Science Foundation. D.K. is supported in part by DOE grant \#DE-FG02-90ER40560.

\footnotetext{
${ }^{2}$ Both in gauge theory and in string theory, in the context of the full configuration of fig. 3 the foregoing discussion is valid for $N_{f} \geq N_{c}$.
} 